\title{
EVOLUTION OF $\boldsymbol{r}$-VALUE DURING THE TENSILE DEFORMATION OF ALUMINIUM
}

\author{
J. SAVOIE, J. J. JONAS, S. R. MACEWEN* and R. PERRIN* \\ Department of Metallurgical Engineering, McGill University, \\ 3450 University Street, Montreal, Canada H3A-2A7
}

(Received 26 December 1994; in final form 28 January 1995)

\begin{abstract}
The elastic strain ratios associated with specific ideal orientations as well as with particular experimental textures were derived from the second order Hill approximation. The single crystal elastic constants for pure aluminum and copper were used. Plastic strain ratios were then calculated for the experimental textures using the FC (full constraint), RC (relaxed constraint) and LW (least work) crystal plasticity codes. Tests were also carried out to determine the $r$-values along various directions in the sheet during Lüders band propagation, as well as the dependence on strain of the plastic $r$-value. It is shown that the observed strain dependence of the $r$-value is related to Lüders band propagation and to the evolution of the texture and not to the elastic-plastic transition.
\end{abstract}

KEY WORDS: Texture, ODF coefficients, elastic anisotropy, plastic anisotropy, $r$-value, Lüders bands.

\section{INTRODUCTION}

It has been commonly observed that the conventional or cumulative plastic strain ratio $r_{p l}$ changes fairly rapidly during the initial stages of straining, finally adopting a near constant value at tensile strains of $15 \%$ or $20 \%$ (Welch et al., 1983; Daniel and Jonas, 1992). Such changes in $r$-value could have their origin in the difference between $r_{e l}$ and $r_{p l}$, where $r_{e l}$ is the elastic strain ratio. However, it has recently been shown (Daniel and Jonas, 1992) that the evolution of $r$-value during the tensile deformation of steels is not primarily associated with the difference between $r_{e l}$ and $r_{p l}$ although it may be affected by the propagation of Lüders bands. Instead, it appears to be a direct consequence of the evolution of the texture during the early stages of tensile deformation. Depending on the actual texture present in the sheet, as well as on the inclination $\theta$ of the axis of the tensile specimen with respect to the initial rolling direction, the texture changes can lead to increases or decreases in $r$-value, as well as to the relative absence of change.

The conclusions described above were based on crystal plasticity calculations and texture measurements carried out on bcc materials and on the occurrence of combined $\{110\}<111>$ and $\{112\}<111>$ slip. Of interest in the present investigation was the extent to which the general phenomenology outlined above applies to fcc metals such as aluminum, in which $\{111\}<110>$ glide is taking place instead. It will be shown below that, while $r_{e l}$ and $r_{p l}$ generally differ in fcc as well as bcc materials, the dependence of $r_{p l}$ on strain observed in samples of aluminum sheets is again related to Lüders band propagation and to texture evolution and not to the elastic-plastic transition.

* Alcan International Limited, Kingston Reasearch and Development Centre, Canada. 


\section{MEASUREMENT OF EXPERIMENTAL $r$-VALUES}

Changes in width and length were measured continuously during the tensile testing of commercial 1145 grade aluminum and of both continuously and batch annealed versions of a 5XXX series experimental alloy. These measurements were carried out at the Alcan KRDC laboratories in Kingston on $190 \mathrm{~mm} \times 19 \mathrm{~mm}$ specimens cut from sheets at several angles with respect to the rolling direction. The samples were then reduced to a constant width of $13 \mathrm{~mm}$ over a length of $100 \mathrm{~mm}$. A double extensometer technique was employed, which allowed the total elongations and width variations to be measured simultaneously ${ }^{1}$. Data acquisition of the load applied to the specimen was also performed; this was used to calculate the stress-strain curves.

As no information about the thickness changes could be collected during straining (due to the specimen geometry), the elastic $r$-value $\left(r_{e l}\right)$ could not be calculated directly from the experimental data. Furthermore, it was not possible to derive the thickness decrease indirectly from the length increase, as this requires knowledge of the volume change during extension. For this reason, in what follows, calculated $r_{e l}$-values are used, as they are known accurately (Daniel and Jonas, 1992).

A further problem arose from the fact that Lüders band propagation takes place rather inhomogeneously as waves of localized deformation pass through the material. Nevertheless, it was possible to measure the average width and thickness strains associated with the Lüders stage of deformation. Their ratio defines an average plastic $r$-value, referred to here as $r_{\text {Luders }}$. Experimental measurements of this ratio were carried out on each specimen of the batch and continuous annealed experimental material by using a single width extensometer. (The thickness strain was determined from the width and length strains in the manner described below.)

The experimental plastic $r$-values $\left(r_{p l}\right)$ were evaluated on the basis of the following definition:

$$
r_{p l}=\frac{\epsilon_{w}}{\epsilon_{t}}
$$

where $\varepsilon_{\mathrm{w}}$ and $\varepsilon_{\mathrm{t}}$ are the plastic strains along the width and thickness directions of the tensile specimen, respectively. The latter were deduced from the relations:

$$
\epsilon_{w}=\ln \frac{w_{f}}{w_{i}} \text { and } \epsilon_{t}=-\left(\epsilon_{l}+\epsilon_{w}\right)
$$

where $w_{i}$ and $w_{f}$ represent the initial (at the beginning of plastic straining) and final (at a defined plastic strain) widths. For this purpose, it is assumed that the specimen volume remains unchanged during plastic straining and that:

$$
\epsilon_{w}=\ln \frac{l_{f}}{l_{i}}
$$

where $l_{i}$ and $l_{f}$ represent the initial and final gauge lengths. (Here a gauge length of $50.8 \mathrm{~mm}$ was used.)

In what follows, two different definitions were used for the plastic $r$-values: the cumulative and the instantaneous. The former is defined by eq. (1), whereas the latter is given by:

\footnotetext{
${ }^{1}$ Instron extensometers of the LVDT type were used. The width extensometer was calibrated to work over the range $10 \mathrm{~mm}-20 \mathrm{~mm}$.
} 


$$
r_{p l}=\frac{\Delta \epsilon_{w}}{\Delta \epsilon_{t}}
$$

where $\Delta \varepsilon_{w}$ and $\Delta \varepsilon_{t}$ represent small simultaneous strain increases. In terms of the $\varepsilon_{w}$ vs $\varepsilon_{t}$ plots presented below, the latter corresponds to the slope at any point, while the former is equivalent to the tangent defined by the coordinate ratio $\varepsilon_{w}: \varepsilon_{t}$.

Stress-strain curves for the $\mathbf{1 1 4 5}$ grade and the continuously annealed experimental alloy, both tested along the rolling direction, are illustrated in Figures 1a and 1b, respectively. The experimental material displays some Lüders elongation followed by the occurrence of dynamic strain aging. The corresponding experimental $\varepsilon_{w}$ vs $\varepsilon_{t}$ curves are presented in Figure 2. The dependence of the $r$-value on elongation strain in the two materials (Figure 3) was calculated from the experimental curves of Figure 2. However, because of the fluctuations in the measured curves of Figure $2 \mathrm{a}$ and the discontinuous yielding associated with the material of Figure $2 b$, the $r$-values determined in this way display oscillations and irregularities. For this reason, a second order polynomial $^{2}$ was fitted to the experimental curves of Figure 2, leading to the smoothed $\varepsilon_{w}$ vs $\varepsilon_{t}$ curves also shown in Figure 2 . These smoothed curves were used to derive the cumulative and instantaneous $r$-value curves presented in Figures 4 and 5 , respectively, for the two classes of material (grade 1145 and the continuous annealed experimental alloy).

The $r$-values pertaining to the 1145 material, shown in Figures 3a, 4a and 5a, change relatively slowly with strain. As will be shown below, these changes are associated with texture evolution. By contrast, the $r$-values associated with the experimental material evolve fairly rapidly with strain, particularly in the first $1.5 \%$ of deformation (see Figures $3 b, 4 b$ and $5 b$ ). These changes are associated with the transition from Lüders band propagation to generalized plastic flow, as will also be seen in more detail below.

\section{EXPERIMENTAL R-VALUES FOR THE PRESENT MATERIALS}

The angular variation in instantaneous $r$-value at the beginning of generalized plastic deformation, determined as described above, was measured in the present three materials. The results are displayed in Figures $6 \mathrm{a}$ to $6 \mathrm{c}$, respectively. All these materials were annealed (i.e. recrystallized). For comparison purposes, the $r$-values corresponding to a cold rolled material, taken from the work of MacEwen et al. (1992), are shown in Figure 6d.

The curve for the first material displays the behaviour identified with the cube texture $\{001\}<100>$ (Figure 6 a): the $r$-value reaches a minimum at $45^{\circ}$ with respect to the rolling direction (RD) and increases progressively from $45^{\circ}$ to $\mathrm{RD}$ and to $\mathrm{TD}$ (the transverse direction). The slight asymmetry can be attributed to the presence of other texture components, such as the Goss $\{011\}<100>$ and the $R\{113\}<575>$ $\left(\varphi_{1}=74^{\circ}, \Phi=31^{\circ}, \varphi_{2}=55^{\circ}\right)$, see below. Similar remarks apply to Figures $6 \mathrm{~b}$ and $6 \mathrm{c}$.

In the case of the cold rolled texture, the $r$-value increases more or less progressively from RD to TD (Figure 6d). This behaviour is explained by the presence (see below)

\footnotetext{
${ }^{2}$ A polynomial function of higher order would have reproduced the strong fluctuations of the curve. These are due to the inherent characteristics of the extensometers used.
} 


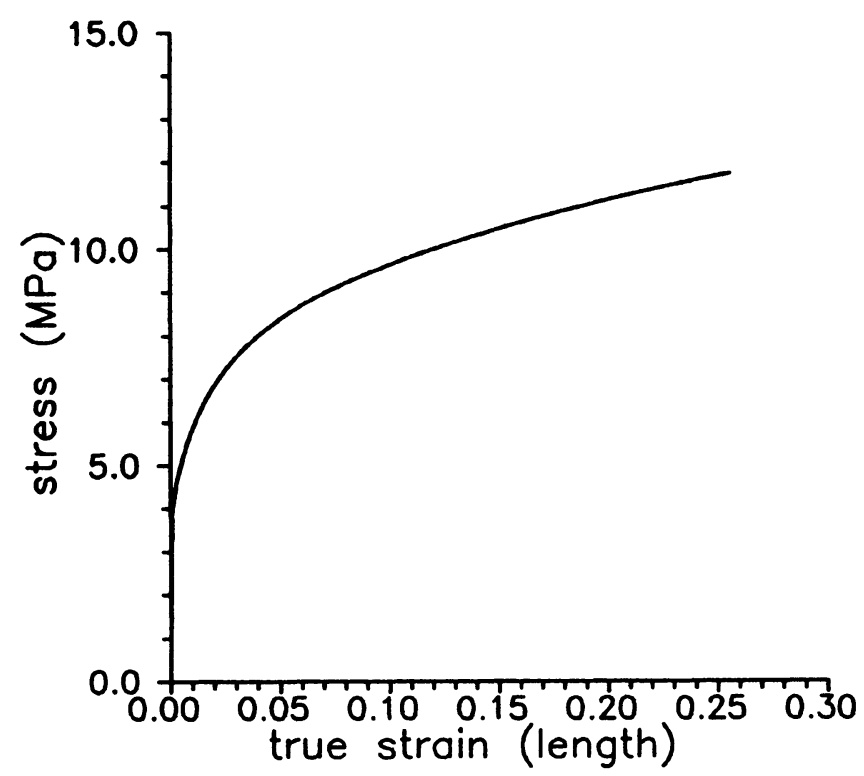

a)

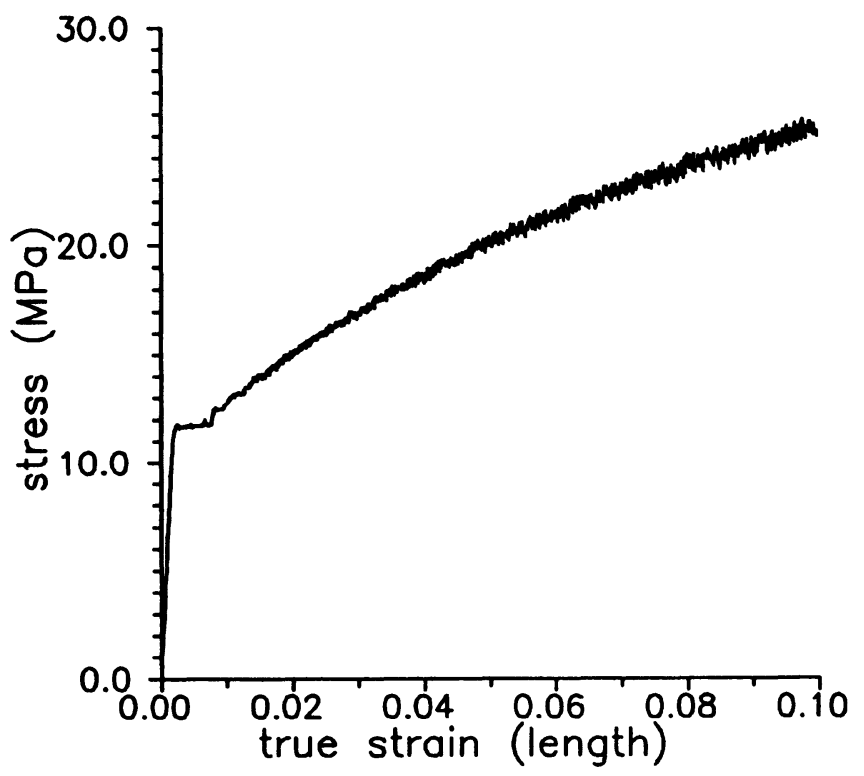

b)

Figure 1 True stress-strain curves for the a) 1145 material tested along RD and b) the continuously annealed experimental alloy tested along RD. 


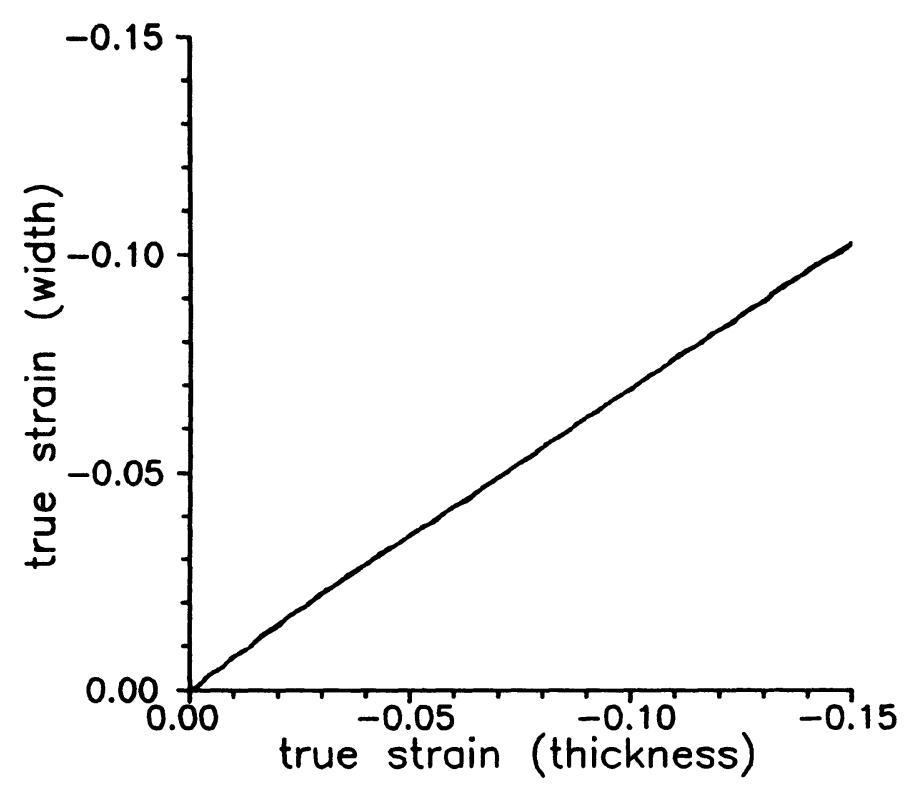

a)

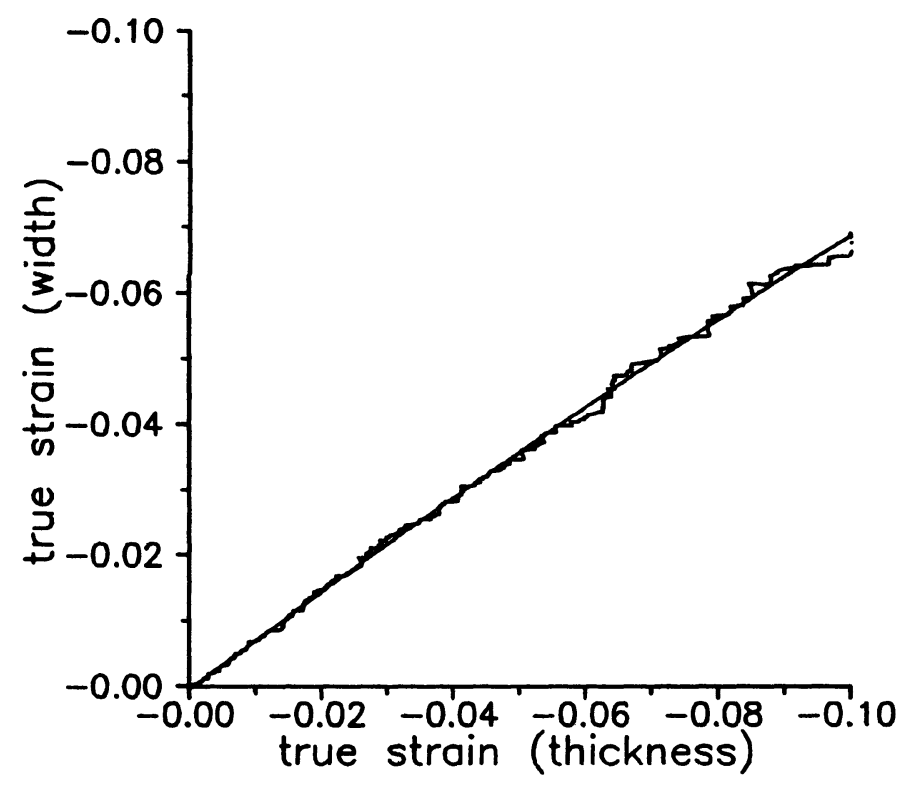

b)

Figure 2 Experimental $\varepsilon_{w}$ vs $\varepsilon_{t}$ curves for a) the 1145 material tested along RD and b) the continuously annealed experimental alloy tested along RD. The polynomial smoothing curves can also be seen. 


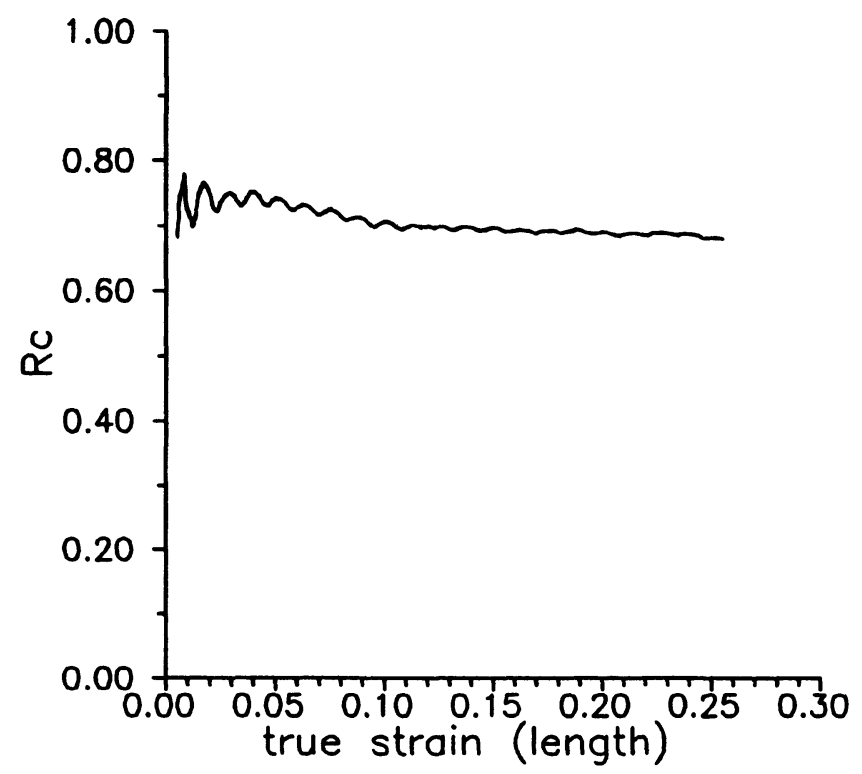

a)

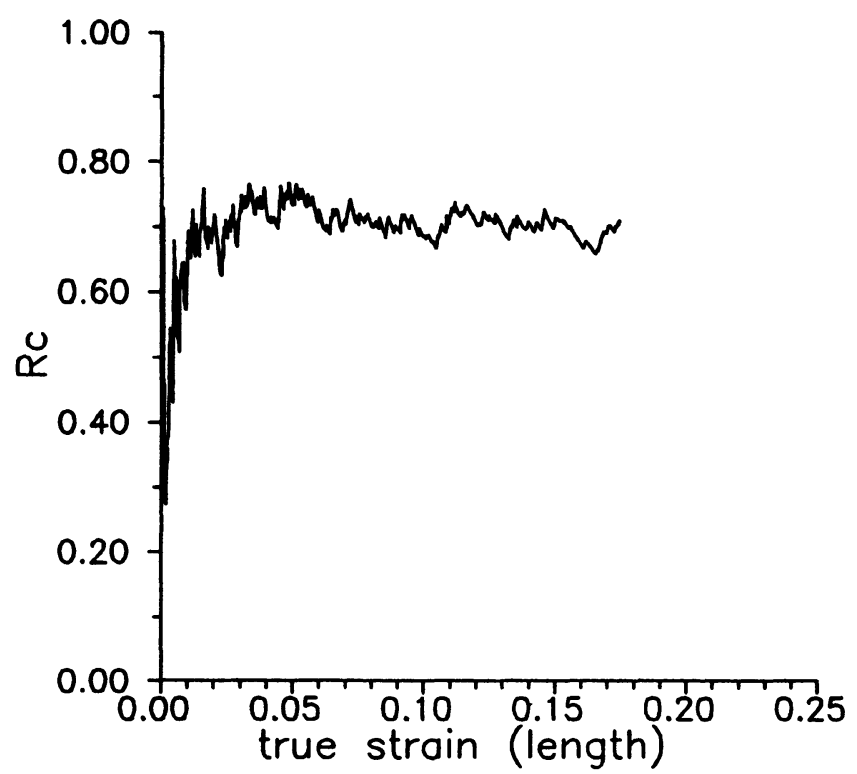

b)

Figure 3 Dependence on elongation strain of the cumulative $r_{p l}$ deduced from the experimental data of Figure 2 for a) the 1145 material tested along RD and b) the continuously annealed experimental alloy tested along RD. 


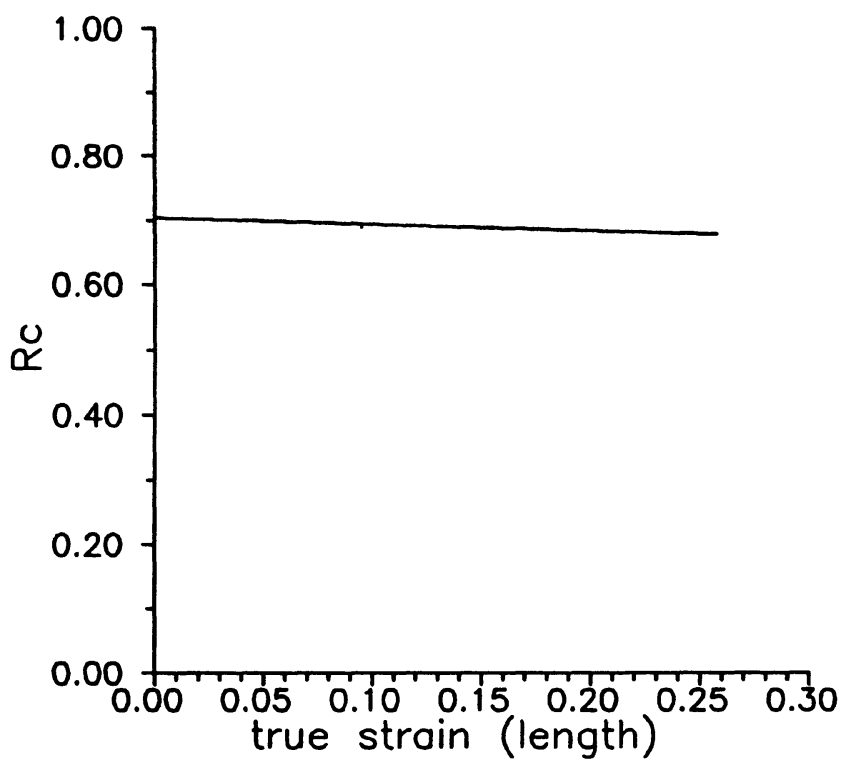

a)

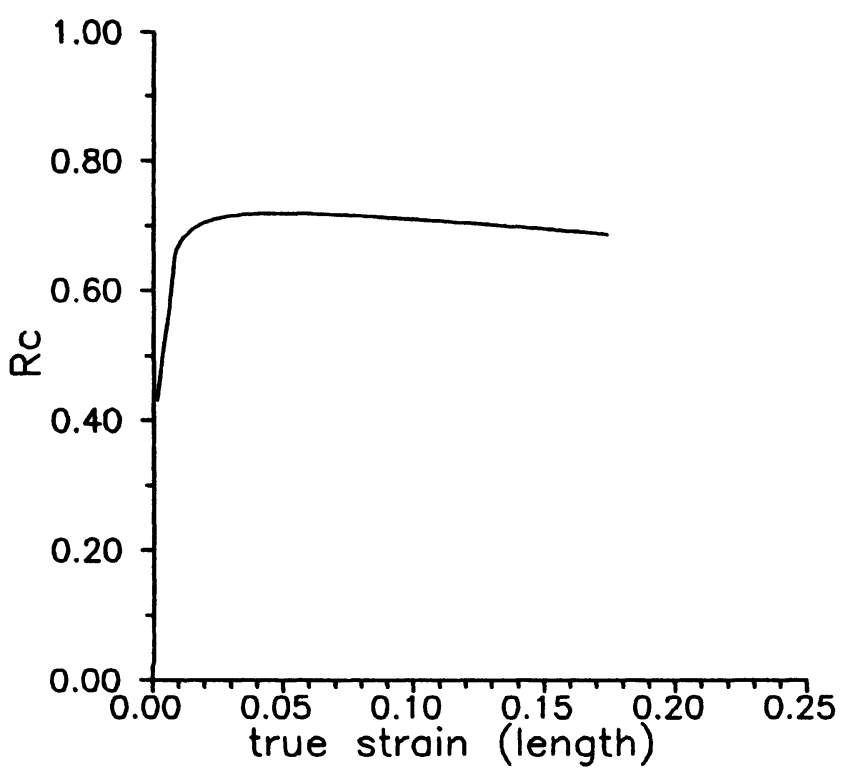

b)

Figure 4 Dependence of the cumulative $r_{p l}$ on elongation strain as determined from the polynomial functions for a) the 1145 material tested along $\mathrm{RD}$ and b) the continuously annealed experimental alloy tested along RD. 


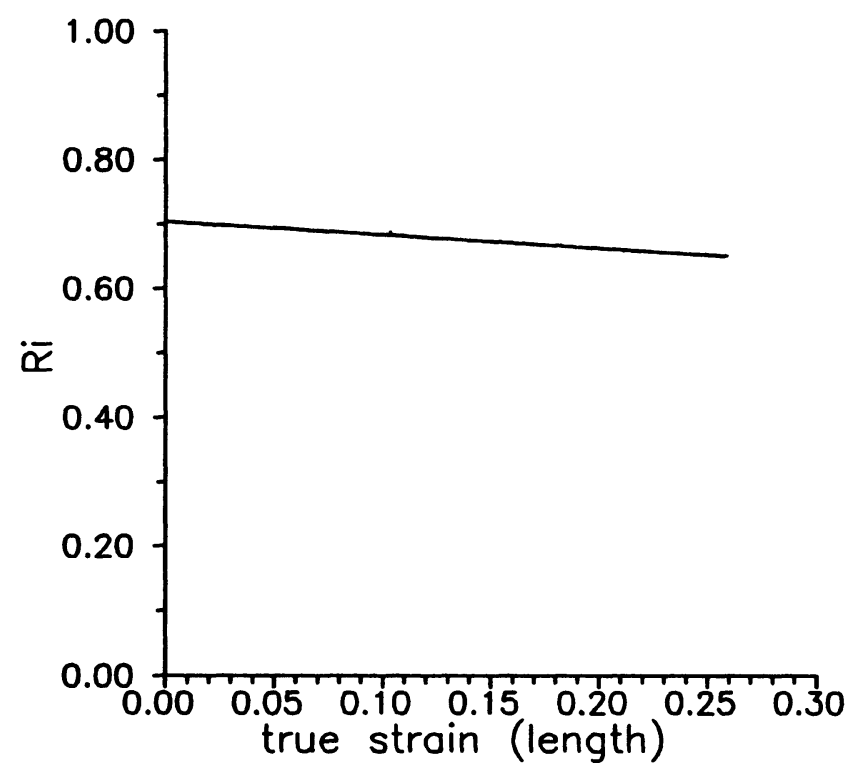

a)

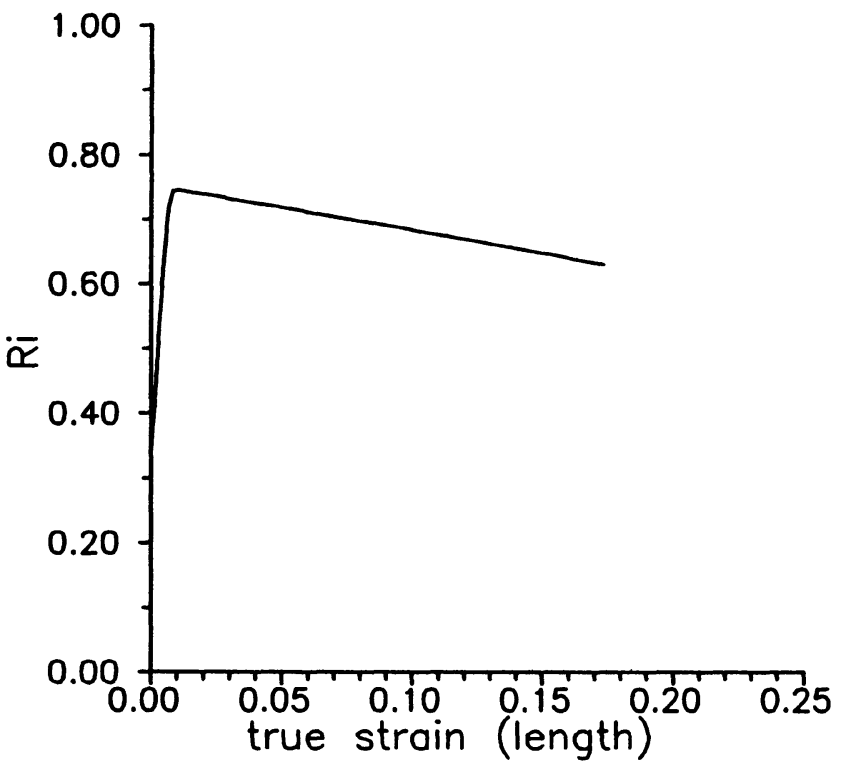

b)

Figure 5 Dependence on elongation strain of the instantaneous $r_{p l}$ determined from the polynomial functions for a) the 1145 material tested along RD and b) the continuously annealed experimental alloy tested along RD. 


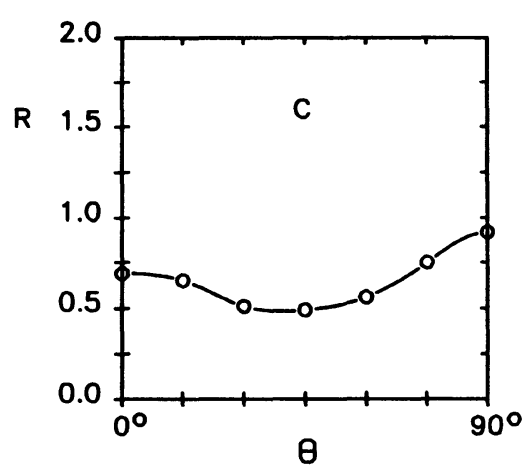

a)

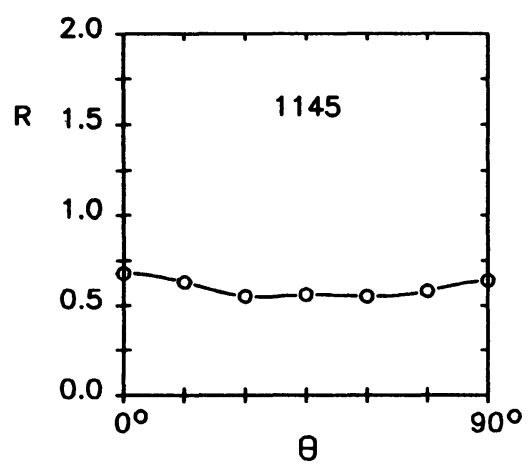

c)

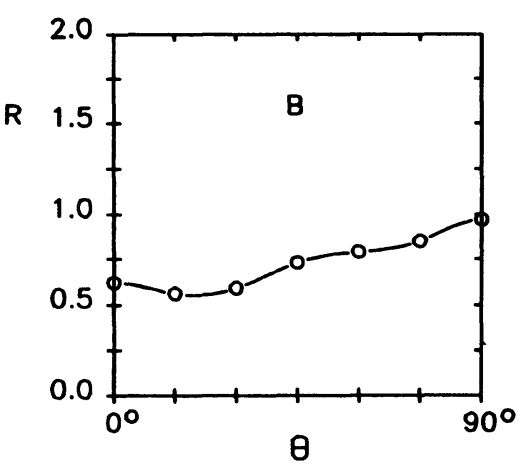

b)

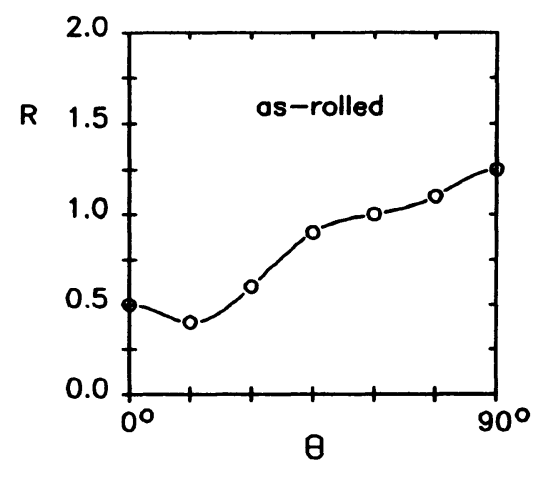

d)

Figure 6 Instantaneous $r$-values determined at the beginning $(\varepsilon=0.025)$ of tensile testing. a) continuously annealed experimental alloy (C), b) batch annealed experimental alloy (B), c) annealed 1145 , and d) as-rolled alloy of reference $/ 2 /$.

of the following rolling components: Goss $\{011\}<100>$, brass or Bs $\{011\}<211>$, $\mathrm{S}\{123\}<634>$, and copper or $\mathrm{Cu}\{112\}<111>$. The influence of each of these texture components will now be considered.

\section{PREDICTION OF $R$-VALUE FROM TEXTURE DATA}

IV.1 Plastic work according to the rate sensitive analysis

The present calculations were carried out using the rate sensitive plasticity approach of Tóth et al. (1988). This involves simulating the tensile deformation of a specimen inclined at an angle $\theta$ to the rolling direction. In the case of very long specimens 
and when rotating grips are used, the macroscopic boundary conditions are the following:

$$
\dot{E}=\left(\begin{array}{ccc}
\dot{E}_{11} & \dot{E}_{12} & 0 \\
\dot{E}_{12} & -\dot{E}_{11} q & 0 \\
0 & 0 & -\dot{E}_{11}(1-q)
\end{array}\right)
$$

where $\dot{E}_{12}$ characterizes the macroscopic shear rate in tension (with $E_{12}=0$ for $\Theta=0^{\circ}$ and $90^{\circ}$ ) and $q$ the contraction ratio $-\dot{E}_{22} / \dot{E}_{11}$, which varies from 0 to 1 . The subscripts 1,2 , and 3 refer to the tensile, width and thickness directions, respectively. The $r$-value is related to $q$ by the equation:

$$
r=\frac{q}{1-q}
$$

In order to calculate the angular variation of $r$ from the texture, several different microscopic conditions can be employed. In the FC (full constraint) case, the shear rates $\dot{\varepsilon}_{i j}$ in each crystallite are prescribed as fully constrained. Some of the shear components are fully relaxed for the RC (relaxed constraint) model, whereas all three are partially relaxed by a factor $\xi_{i j}$ for the present LW (least work) model (Engler et al., 1993). (Here $\dot{\varepsilon}_{i j}=\xi_{i j} \cdot \dot{\varepsilon}_{i j}^{0}$, with $0<\xi<1$, and $\dot{\varepsilon}^{0}$ as the fully relaxed value of the shear rate component under consideration). Accordingly, the following microscopic strain rate tensor $\dot{\varepsilon}$ was used (expressed here in the rolling coordinate system, where the subscripts 1, 2 and 3 now refer to the rolling, transverse and normal directions, respectively). The above coordinate system coincides with the tensile reference frame for $\theta=0^{\circ}$.

$$
\dot{\boldsymbol{\epsilon}}=\left(\begin{array}{ccc}
\dot{\epsilon}_{11} & \xi_{12} \cdot \dot{\epsilon}_{12}^{0} & \xi_{13} \cdot \dot{\epsilon}_{13}^{0} \\
\xi_{12} \cdot \dot{\epsilon}_{12}^{0} & -\dot{\epsilon}_{11} q & \xi_{23} \cdot \dot{\epsilon}_{23}^{0} \\
\xi_{13} \cdot \dot{\epsilon}_{13}^{0} & \xi_{23} \cdot \dot{\epsilon}_{23}^{0} & -\dot{\epsilon}_{11}(1-q)
\end{array}\right)
$$

For an imposed value of $q$ and a given grain orientation $g$, the local plastic work rate $\dot{W}_{q}(g)$ (per unit volume) was calculated using the FC, one RC (pancake, $\dot{\varepsilon}_{13}$ and $\dot{\varepsilon}_{23}$ fully relaxed) and LW deformation modes. Here:

$$
\dot{W}_{q}(\dot{g})=\sum_{s=1}^{s_{\max }} \dot{\gamma}^{s} \cdot \tau^{s}
$$

where $\gamma_{s}$ and $\tau_{s}$ are the shear rates and shear stresses, respectively, that apply to slip system $s$. A peculiarity of the rate sensitive model is that the $\tau^{s}$ values (which differ from $\tau_{0}$ ) vary from one slip system to another, especially for high values of $m$. It is not therefore possible to calculate the plastic work rate per unit CRSS (or $\tau_{0}$ ) using solely the sum of the crystallographic shears, as in rate insensitive models. The local plastic work rate must thus be defined as the sum of the crystallographic shears multiplied by the resolved shear stress $\tau^{\text {s }}$ pertaining to each slip system.

The LW model used here is similar to that proposed in a previous paper (Engler et al., 1993), where a range of values is employed for $\xi_{i j}$, the degree of relaxation. of each shear rate $\dot{\varepsilon}_{i j}$. In this model, the $\xi_{i j}$ are determined from the differences $\Delta \dot{W}$ in deformation energy between the full constraint case and the special type of RC case in which all three shear rate components are relaxed. For this purpose, a shear capacity 
$\alpha_{i j}$ is defined as the ratio of the energy saved by relaxing the constraints to the amount of the fully relaxed shear:

$$
\alpha_{i j}=\frac{\Delta \dot{W}}{\left|\dot{\epsilon}_{i j}^{0}\right|}=\frac{\dot{W}_{q}^{F C}-\dot{W}_{q}^{R C}}{\left|\dot{\epsilon}_{i j}^{0}\right|}
$$

An orientation associated with a shear component $\dot{\varepsilon}_{i j}$ that leads to a large energy reduction will be associated with a higher value of $\xi_{i j}$ than an orientation with a small reduction of energy. $\xi_{i j}$ is then calculated from:

$$
\xi_{i j}=\frac{\alpha_{i j}}{\alpha_{i j}^{\max }}
$$

where $\alpha_{i j}^{\max }$ is defined, for simplicity, as the maximum shear capacity for all possible orientations in orientation space.

\section{IV.2 Plastic Strain Ratio}

For a polycrystalline sample, the macroscopic rate of plastic work corresponding to a given $q$ value is given by the relations in Bunge $(1970 ; 1982)$. However, such calculations are based on the Taylor factor $M_{q}(g)$ instead of on the rate of plastic work $\dot{W}_{q}(g)$ defined above. For this reason, the following relations, where $M$ is replaced by $\dot{W}$, were used. Various $q$-responses of the material were studied. As in the Taylor factor approach, the value of $q$ that minimizes the macroscopic deformation energy was selected. Using the series expansion formalism, the rate of plastic work for a given texture and value of $q$ is then defined as:

$$
{\overline{\dot{W}_{q}}}_{q}=\sum_{l=0}^{L_{\max }} \sum_{\mu=1}^{M(l)} \sum_{\nu=1}^{N(l)} \frac{w_{l}^{\mu \nu}(q) \cdot C_{l}^{\mu \nu}}{2 l+1}
$$

where $w^{\mu \nu}{ }_{l}$ are the series expansion coefficients of the plastic work rates integrated throughout Euler space (Bunge, 1982). The $C^{\mu \nu}{ }_{1}$ coefficients were determined from the initial sheet textures by means of $\mathrm{X}$-ray measurements, using standard techniques (see below).

Bunge (1982) has shown that the mean Taylor factor calculated along the lines of equation (11) can be used to derive the energy dissipated when the strain rate tensor is expressed in the specimen coordinate system as opposed to the one in which the ODF is described (i.e. the rolling reference frame). As the normal direction in the rolling reference frame coincides with the thickness axis of the sample coordinate system, the above relation becomes:

$$
\overline{\dot{W}}_{q}(\theta)=\sum_{l \mu \nu} \frac{C_{l}^{\mu \nu} \cdot w_{l}^{\mu \nu}}{2 l+1} \cdot \cos (2(\nu-1) \theta)
$$

To predict $r$-values from the present experimental ODF's, a data bank of $w_{l}^{\mu \nu}$ coefficients was prepared corresponding to several deformation modes. This was carried out in the rolling reference frame, for which $\Theta=0^{\circ}$. For non-zero values of $\theta, r_{p l}$ was found by minimizing $\dot{W}_{q}(\Theta)$ with the aid of a polynomial regression. The $r$-value obtained in this way corresponds to the instantaneous value associated with a given texture rather than the average or cumulative $r$-value conventionally measured at 15 or $20 \%$ strain. 


\section{IV.3 Texture measurements}

For the texture measurements, four incomplete pole figures: $\{111\},\{002\},\{022\}$ and \{113\} (with $5^{\circ} \leq \alpha \leq 80^{\circ}$ ) were measured using a Siemens texture goniometer. After correction of the pole figure data, the ODF's were calculated using the series expansion method, leading to the determination of the $C_{1}^{\mu \nu}$ coefficients up to $l=22$ (Bunge, 1982). For the reduction of ghost effects, the exponential method was used in order to determine the odd terms of the series expansions (Van Houtte, 1991).

\section{IV.4 Plastic r-values of single texture components}

The angular variation of $r$-value was calculated using the pancake model for five single texture components. (The latter led to the best agreement with the experimental data, see section V). The ODF coefficients associated with each of these ideal orientations were calculated using a gaussian scatter width of $\omega_{0}=8^{\circ}$ and a volume fraction $M_{i}=100 \%$. This type of gaussian distribution was used to account for the misorientation of individual grains with respect to the ideal orientation. The results are presented in Figure 7.

It is clear from Figure 7a, that the cube (recrystallization) component is responsible for the angular variation in $r$-value displayed in Figure 6a. The secondary components, such as the Goss and $R$ (Figures $7 b$ and $7 c$, respectively), disturb the symmetry associated with the cube texture: the presence of the Goss increases the value of $r$ at $90^{\circ}$, while that of the $\mathrm{R}$ increases $r$ at approximately $45^{\circ}$.

The calculated $r$-values corresponding to the main rolling texture components are displayed in Figures $7 \mathrm{~b}$ to $7 \mathrm{e}$ for the Goss, $\mathrm{S}^{3}, \mathrm{Bs}$ and $\mathrm{Cu}$, respectively. Here it can be seen that there is a marked maximum at $45^{\circ}$. However, the maximum $r$-value in the rolled material of Figure $6 \mathrm{~d}$ is reached at $90^{\circ}$. This can be attributed to the presence of the Goss component in the rolling texture (see Figure $7 b$ ).

\section{COMPARISON BETWEEN EXPERIMENTAL DATA AND MODELLING PREDICTIONS}

The experimental $r$-values displayed in Figure 6 (for $\varepsilon=0.025$ ) are compared with the modelling data obtained from the FC and pancake models in Figure 8 . The initial texture coefficients of the sheet were employed in these calculations. For these materials, the FC mode does not reproduce the experimental data as well as the predictions of the pancake model. In the as-rolled material, the FC model leads to large deviations around $45^{\circ}$; these discrepancies are consistent with the elongated grain shapes observed in the rolled samples.

The use of the least work model leads to a compromise between the predictions of the pancake and FC models. Some calculations carried out in this way are presented in Figure 9. Here it can be seen that the predictions for the three annealed materials are in reasonable agreement with the observations and are about as accurate as those of the pancake model in Figure 8. For the as-rolled material, however, only the pancake predictions fit the experimental data in a reasonable way.

\footnotetext{
${ }^{3}$ Note that the $R\{113\}<575>$ and $S\{123\}<634>$ components are not considered here as distinct as they lie close to each other and display similar calculated angular variations in $r$-value.
} 


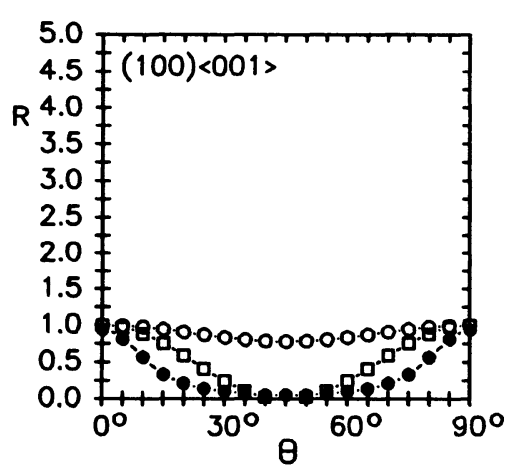

a)

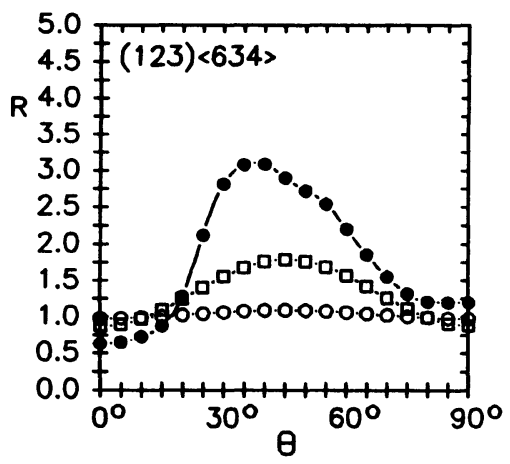

c)

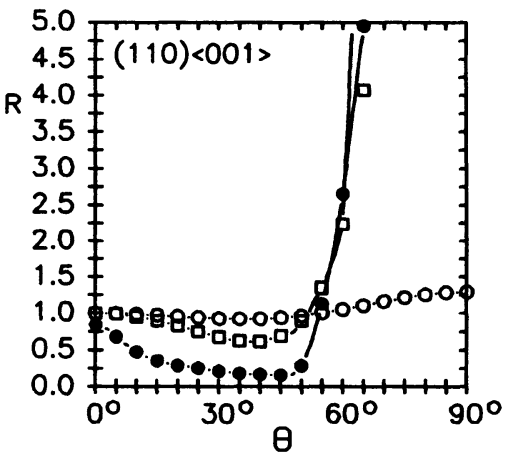

b)

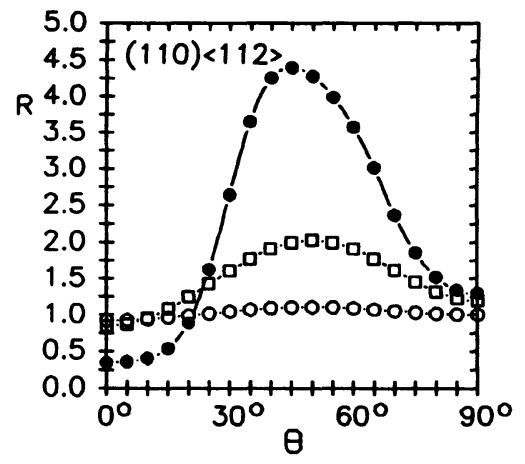

d)

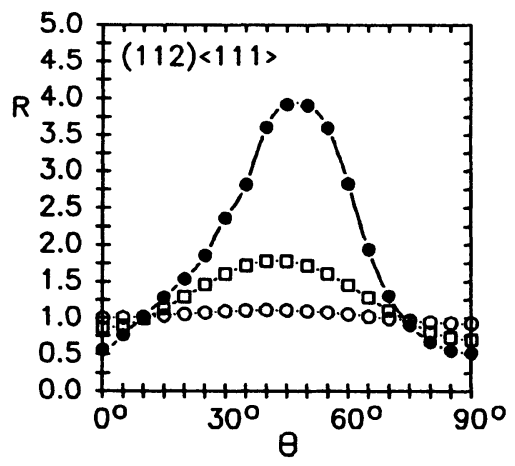

e)

Figure 7 Predictions of $r_{p l}(\Theta)$ using the pancake model $(\bullet)$ and of $r_{e l}(\Theta)$ for $\mathrm{A} 1(\odot)$ and $\mathrm{Cu}(\square)$ using the second order Hill approximation. Figures $7 \mathrm{a}$ to $7 \mathrm{c}$ display the behaviours of three recrystallization components, while Figures $7 \mathrm{~b}$ to $7 \mathrm{e}$ illustrate those of the four rolling texture components. The Goss $\{110\}<110>$ and $S / R\{123\}<634>$ orientations (Figures $7 b$ and $7 c$ ) are present in both recrystallization and rolling textures. 


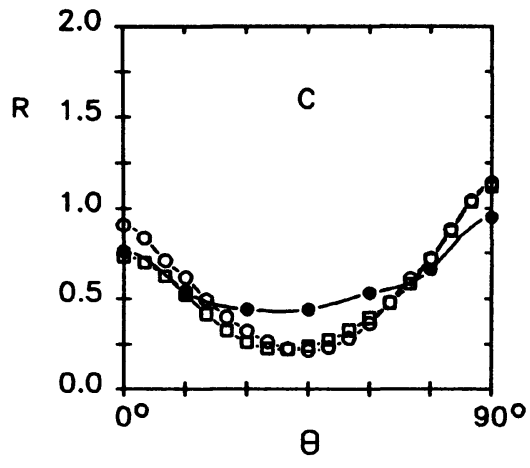

a)

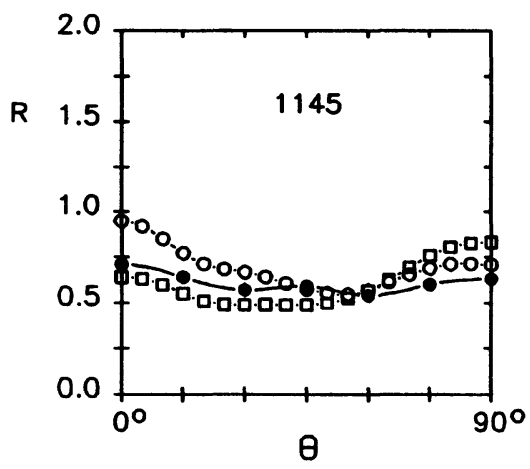

c)

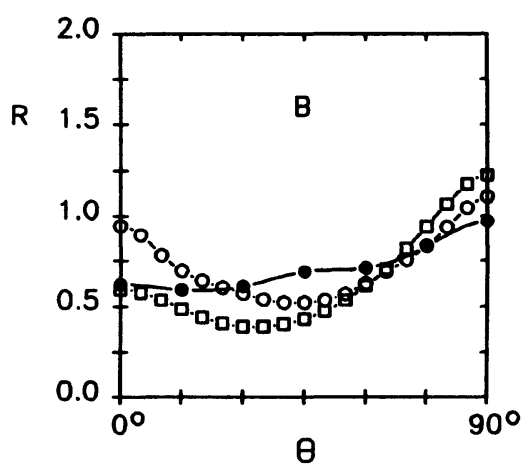

b)

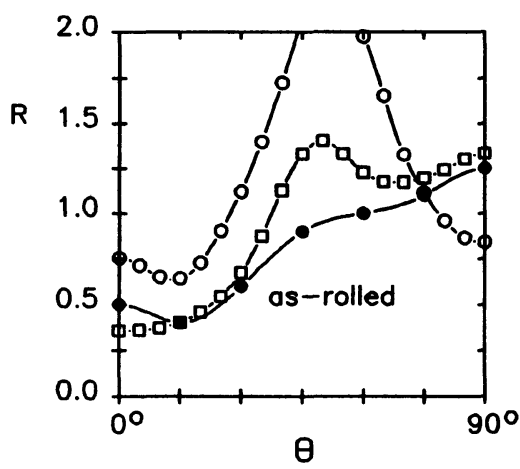

d)

Figure 8 Predictions of $r_{p l}(\Theta)$ using the FC $(O)$ and pancake $(\square)$ models. The experimental data (O) are also shown: a) continuously annealed experimental alloy (C), b) batch annealed experimental alloy (B), c) annealed 1145 and d) as-rolled alloy of reference $/ 2 /$.

\section{EFFECT OF TEXTURE EVOLUTION ON $R$-VALUE}

The textures of selected specimens were measured after tensile deformation: along RD alone for the 1145 material and continuously annealed experimental alloy, and along directions taken at intervals of $15^{\circ}$ between RD and TD for the batch annealed experimental alloy. In the latter case, the sample symmetry is monoclinic. The ODF's measured before and after tensile deformation are shown in Figure 10 for the batch annealed experimental alloy. Here the tensile specimen was aligned along RD. The results obtained for specimens aligned at an angle of $60^{\circ}$ with respect to $\mathrm{RD}$ are presented in Figure 11. It can be seen from these ODF's that the textures changed significantly during plastic deformation (to $\varepsilon=0.15-0.20$ ). These texture changes can be expected to contribute to $r$-value evolution during tensile testing. 


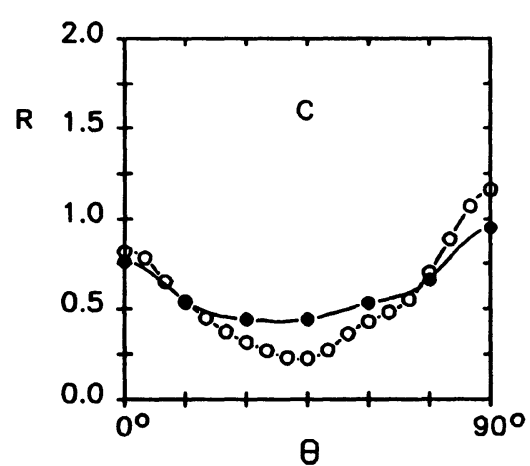

a)

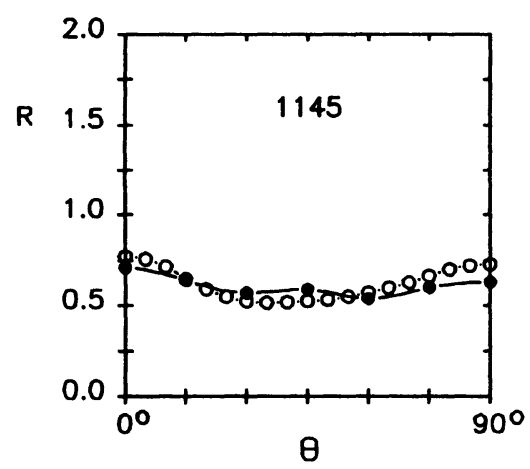

c)

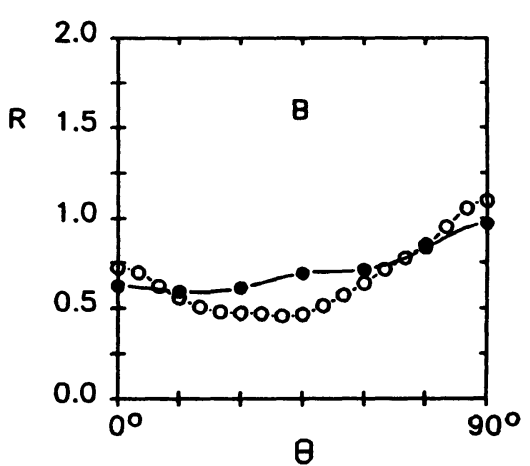

b)

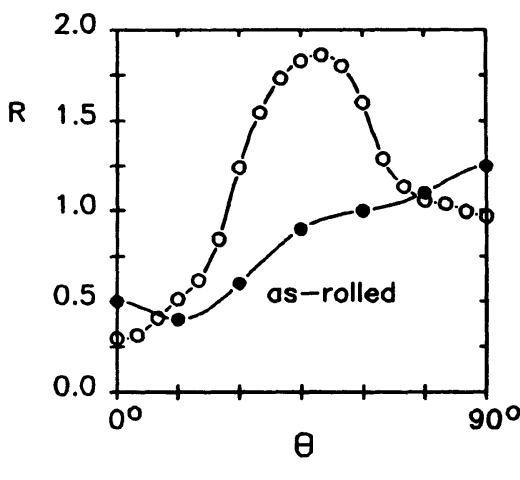

d)

Figure 9 Predictions of $r_{p l}(\theta)$ using the LW $(O)$ model. The experimental data $(O)$ are also shown: a) continuously annealed experimental alloy (C), b) batch annealed experimental alloy (b), c) annealed 1145 and d) as-rolled alloy of reference $/ 2 /$.

In order to test the magnitude of this effect, $r$-values were calculated using the texture coefficients determined before and after tensile straining. Then these were compared with the corresponding instantaneous experimental $r$ 's. The results are summarized in Table 1 in the form of the changes in $r, \delta r$, taking place during testing or predicted from the texture data using the pancake model. For seven of the eight samples, the change in $r$-value during straining can be related directly to texture evolution, as the predicted increases or decreases correspond approximately to the experimental ones (within a $\delta r$ difference of 0.05 ). However, in one case, the changes are of opposite sign and differ by 0.10 . 


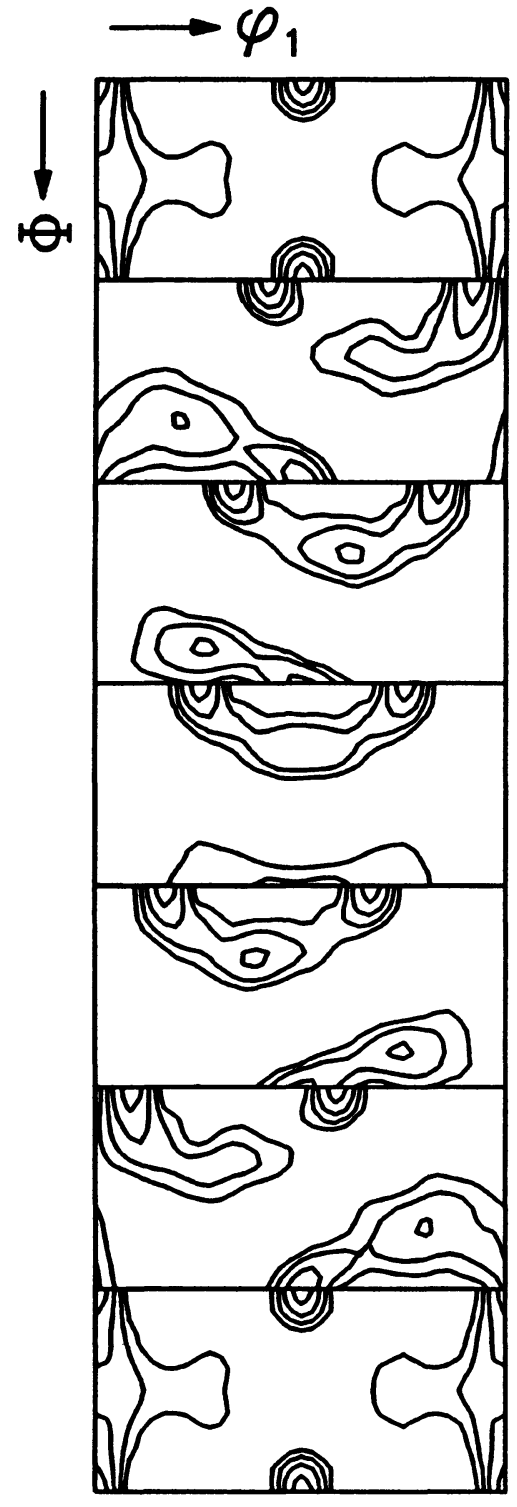

a)

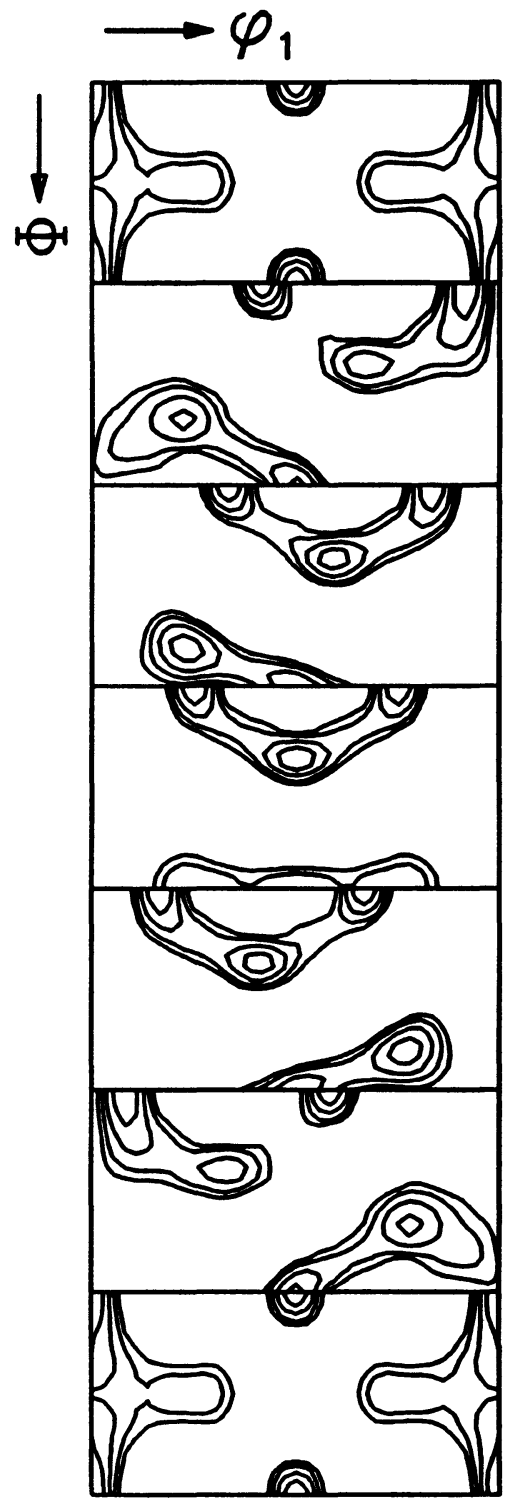

b)

Figure 10 ODF's of the batch annealed experimental alloy determined a) before and b) after tensile deformation $(\varepsilon=0.15-0.20)$ along RD. $\varphi_{2}$ sections $\left(0^{\circ}-90^{\circ}, 15^{\circ} \times 15^{\circ}\right)$. Levels: $1-2-4-7$ 


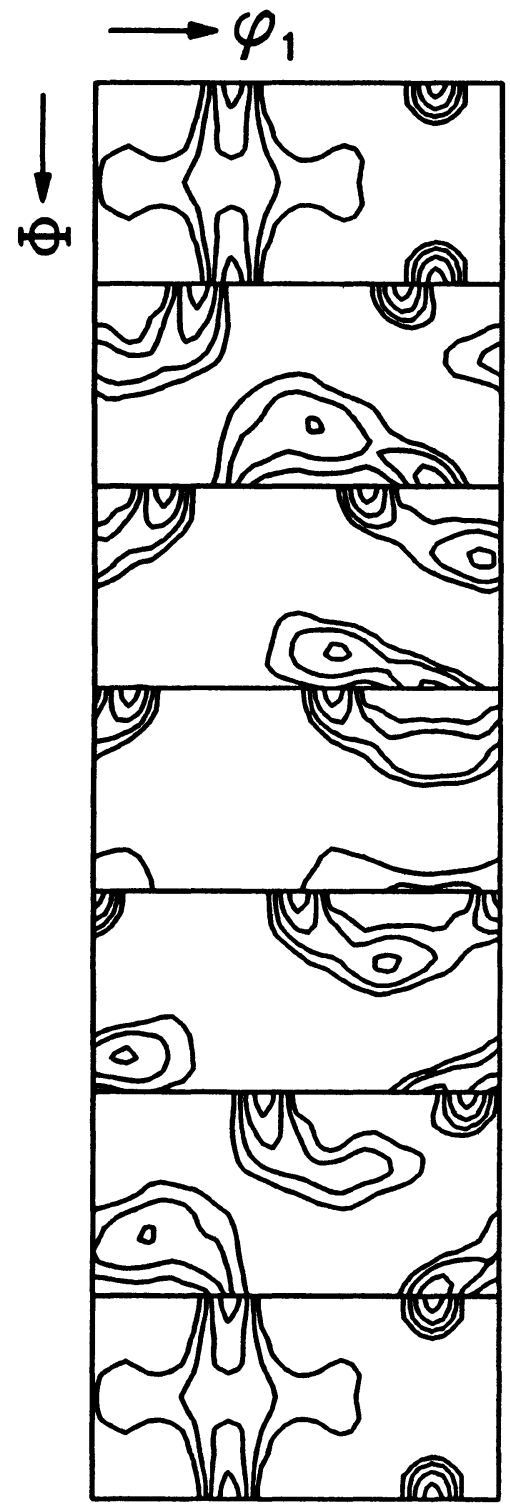

a)

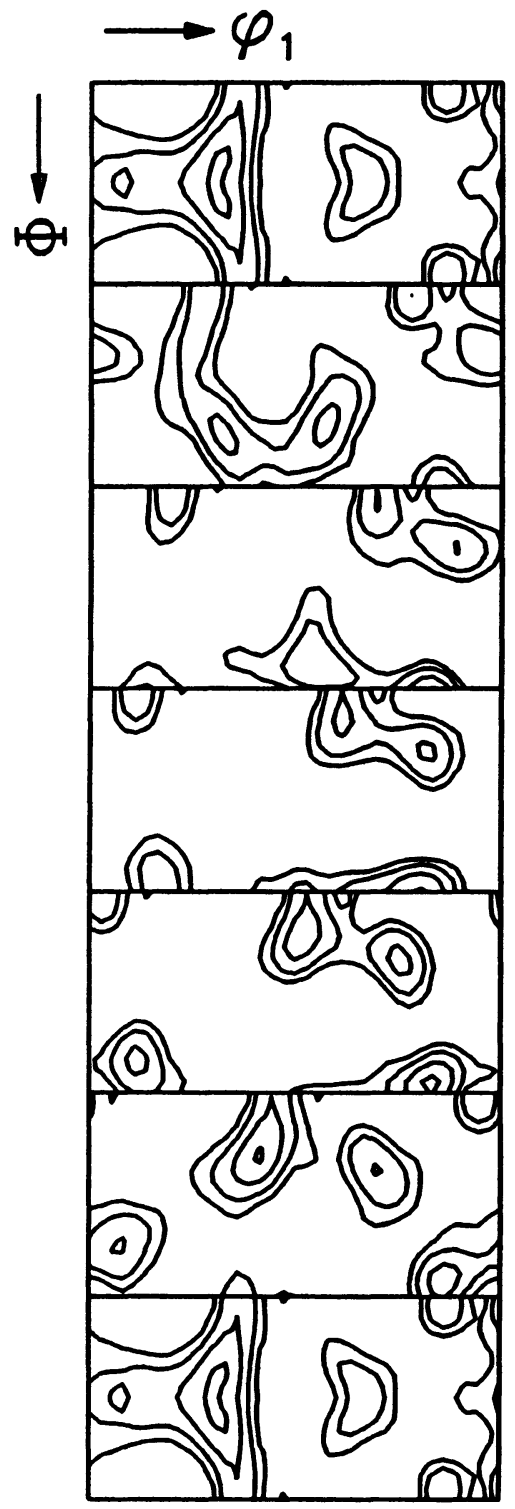

b)

Figure 11 ODF's of the batch annealed experimental alloy determined a) before and b) after tensile deformation $(\varepsilon=0.15-0.20)$ of samples with axes inclined at $60^{\circ}$ to $\mathrm{RD}$. $\varphi_{2}$ sections $\left(0^{\circ}-90^{\circ}\right.$, $\left.15^{\circ} \times 15^{\circ}\right)$. Levels: $1-2-4-7$ 
Table 1 Comparison of experimentally measured and predicted $\delta r$ 's.

\begin{tabular}{ccc}
\hline Sample & $\delta r_{\text {experiment }}$ & $\delta r_{\text {prodiction }}$ \\
1145-RD & -.06 & -0.2 \\
C X-RD & -.13 & -.08 \\
B X-15 & -.04 & .00 \\
B X-30 & -.03 & -.03 \\
B X-45 & .06 & .02 \\
B X-60 & .13 & .07 \\
B X-75 & .03 & .04 \\
B X-TD & -.03 & .07 \\
\hline
\end{tabular}

\footnotetext{
$\mathrm{C}=$ continuously annealed

$\mathrm{B}=$ batch annealed

$\mathrm{X}=$ experimental alloy
}

\section{ELASTIC $R$-VALUES AND THE ELASTIC-PLASTIC TRANSITION}

\section{VII.1 Planar variation of $r_{e l}$ and $r_{p l}$}

The angular variation of the elastic and plastic strain ratios pertaining to the main ideal orientations observed experimentally in fcc sheets was presented in Figure 7. For this purpose, $r_{e l}(\Theta)$ was calculated using the second order Hill approximation (Bunge, 1974). The dependence of Young's modulus on $\Theta$ was also calculated using the elasticity model (Figure 12). As aluminum displays little elastic anisotropy due to its unique elastic properties, similar calculations were also carried out using the elastic constants of pure copper (Table 2). Given that, in the model calculations, the plastic anisotropy depends only on the texture, the $r_{p l}$ predictions for aluminum and copper are identical. By contrast, the elastic data for the copper display much stronger elastic anisotropy than those for the aluminum, although the two materials follow the same tendencies.

As expected, $E(\Theta)$ and $r_{e l}(\theta)$ display opposite dependences on $\Theta$. The copper $r_{e l}$ curves follow the general tendencies of the $r_{p l}(\Theta)$ curves, even though it was shown above that $r_{p l}$ and $r_{e l}$ are not strictly proportional along a given $\boldsymbol{\theta}$ direction. The aluminum $r_{e l}$-values remain close to 1 ; thus $r_{e l}$ is greater or less than $r_{p l}$ depending on whether the latter is less than or greater than 1 , respectively. The elastic-plastic transition in $r$-value is thus expected to involve a rapid change from a value close to 1 to $r_{p l}$ as soon as plastic deformation is initiated (see Figure 13).

\section{VII.2 Transition from Lüders band propagation to generalized plastic flow}

Experimental measurements of the $r_{L u ̈ d e r}$-values were carried out on each specimen of the batch and continuous annealed experimental material, as explained in section II. In the batch annealed material, the average value of $r_{\text {Luders }}$ determined in the $\theta$ range $0^{\circ}$ to $45^{\circ}$, inclusive, was 0.5 , whereas it was 1.3 over the $\theta$ range $60^{\circ}$ to $90^{\circ}$. For the continuous annealed material, the average over the $\Theta$ range $0^{\circ}$ to $60^{\circ}$, inclusive, was 0.4 , whereas it was 1.0 over the $75^{\circ}$ to $90^{\circ}$ interval (Table 3). It thus appears that the measured $r_{\text {Liders }}$-values for both experimental materials differ distinctly from 


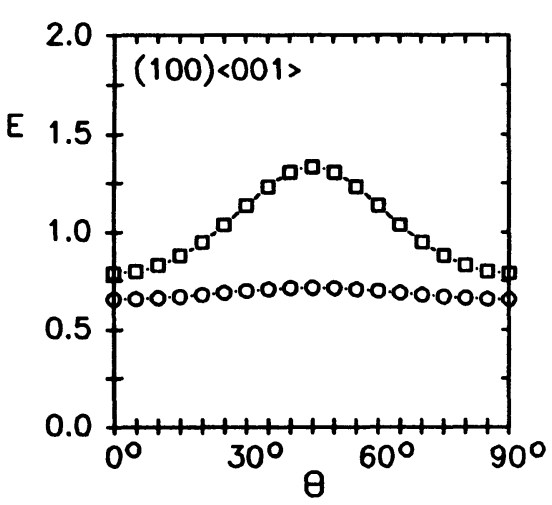

a)

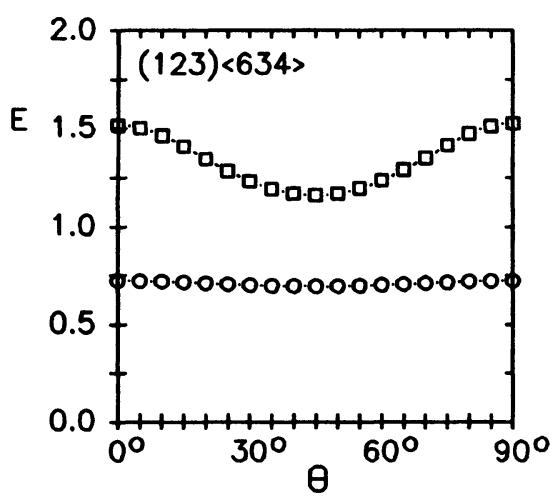

c)

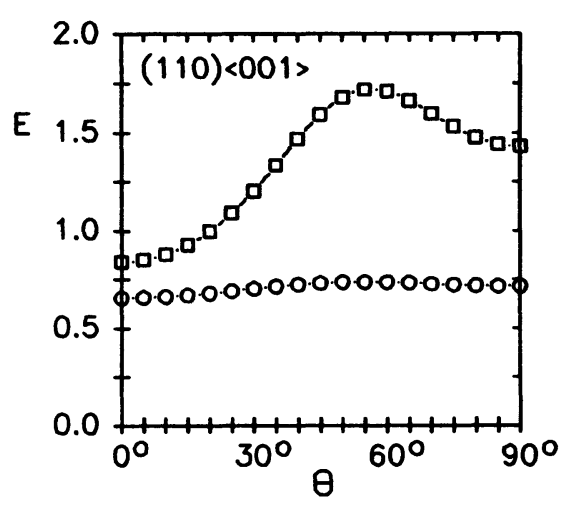

b)

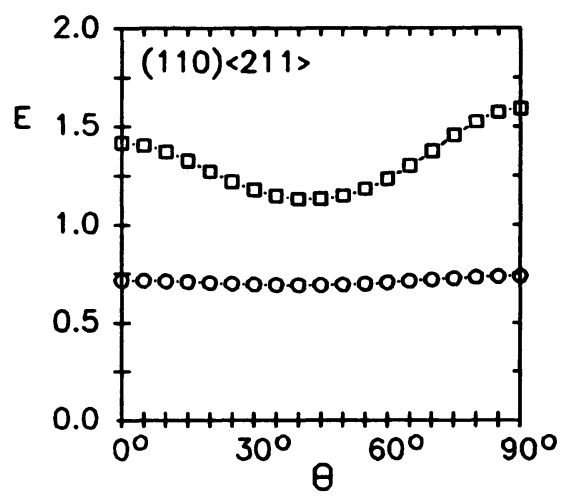

d)

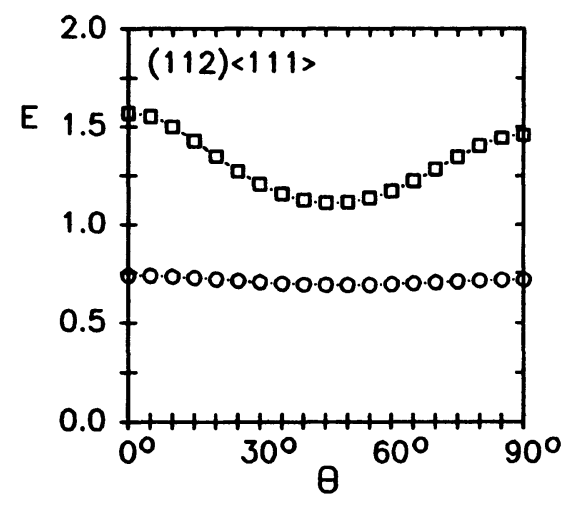

e)

Figure 12 Predictions of $E(\theta)$ for $\mathrm{A} 1(\mathrm{O})$ and $\mathrm{Cu}(\mathrm{C})$ using the second order Hill approximation for the ideal orientations of Figure 7. 
Table 2 Elastic constants of $\mathrm{Al}$ and $\mathrm{Cu}$.

\begin{tabular}{cccc}
\hline Material & $\mathrm{C}_{11}$ & $\mathrm{C}_{12}$ & $\mathrm{C}_{44}$ \\
$\mathrm{AI}$ & 1.130 & 0.666 & 0.278 \\
$\mathrm{Cu}$ & 1.688 & 1.216 & 0.757 \\
\hline
\end{tabular}

those determined after conventional flow has begun (see Figures $6 a$ and $6 \mathrm{~b}$ ).

Let $\alpha$ represent the inclination of the Lüders front to the tensile axis. Then the value $\alpha_{i}=54.7^{\circ}$ applies to isotropic materials $(r=1)$, while $\alpha$ is greater than $\alpha_{i}$ for the first $\theta$ range (e.g. $\alpha=64.3^{\circ}$ for $r=0.3$ ) and less than $\alpha_{i}$ for the second $\theta$ range (e.g. $\alpha=52.2^{\circ}$ for $r=1.5$ ) (see Appendix). Thus, tensile testing over the $\theta$ range $0^{\circ}$ to $45^{\circ}$ or $0^{\circ}$ to $60^{\circ}$, which led to $r_{\text {Luders }}$ values below one (more thickness decrease than width decrease) seems to involve Lüders fronts with higher inclinations with respect to the tensile axis; by contrast, testing over the $\theta$ range $60^{\circ}$ to $90^{\circ}$, which led to $r_{\text {Luders }}$ values above one (i.e. more width decrease than thickness decrease) appears to be associated with Lüders front inclinations that are closer to $45^{\circ}$.

It thus appears that, in the experimental alloy, the $r$-value changes suddenly during the transition from Lüders band propagation to generalized plastic flow. By contrast, during tensile testing of the 1145 alloy, in which no Lüders bands are observed, the transition between $r_{e l}$ and $r_{p l}$ is much smoother, although both materials display the same kind of angular dependence of $r$-value on angle $\theta$. Schematic illustrations of the expected strain dependences of $r$ in the cases of homogeneous and inhomogeneous yielding are presented in Figure 13.

\section{CONCLUSIONS}

1. The angular variations in $r$-value (with respect to the rolling direction) were determined from experimental data using the instantaneous definition of $r$. The variations in the annealed materials were consistent with the cube texture being dominant. The $r$-value profile in the as-rolled material was consistent with the presence of cold rolling texture components.

2. The angular variation in $r$-value of the annealed materials can be reproduced very accurately using both the pancake and least work deformation modes. However, the as-rolled material data could only be reproduced with the pancake mode. This behaviour is consistent with the presence of pancaked grains after rolling.

3. The plastic $r$-value changes with strain when both the instantaneous and cumulative definitions of $r$ are used. These changes can be attributed to the evolution of the texture during deformation.

4. The elastic-plastic transition in $r$-value displays a rapid change from a value close to 1 (for aluminum) to $r_{p l}$ as soon as plastic deformation is initiated. When Lüders band propagation occurs, the $r$-value changes suddenly during the transition from Lüders band generalized plastic flow. These changes can involve rapid increases or decreases in $r$-value. By contrast, for materials in which no Lüdres bands are observed, the transition between $r_{e l}$ and $r_{p l}$ is much smoother. 


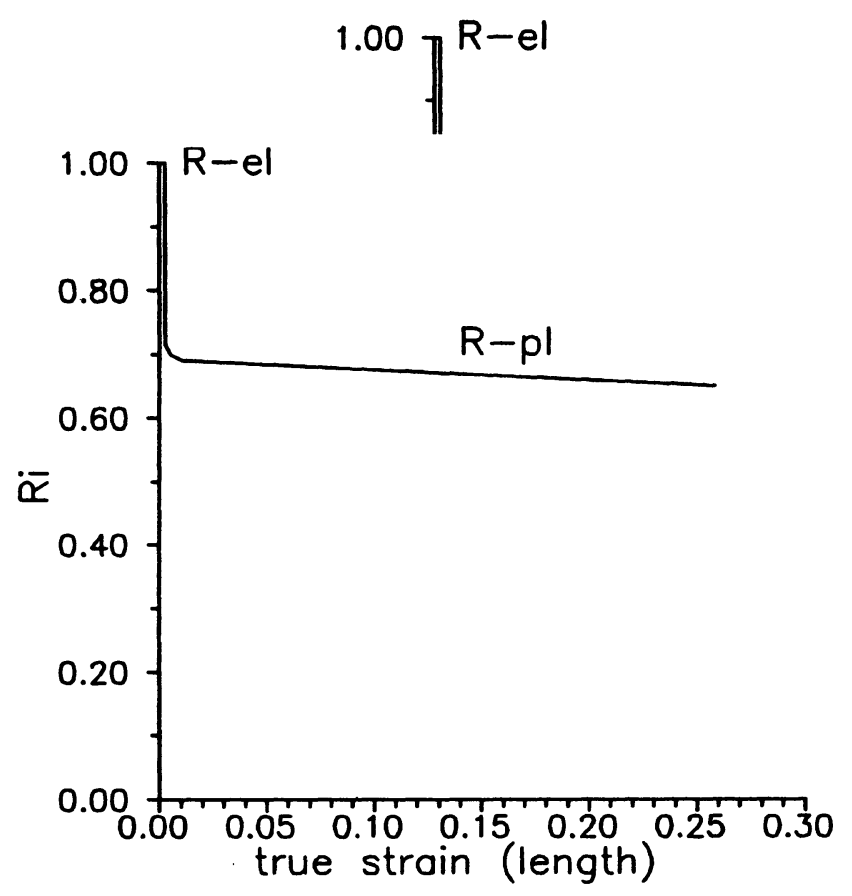

a)

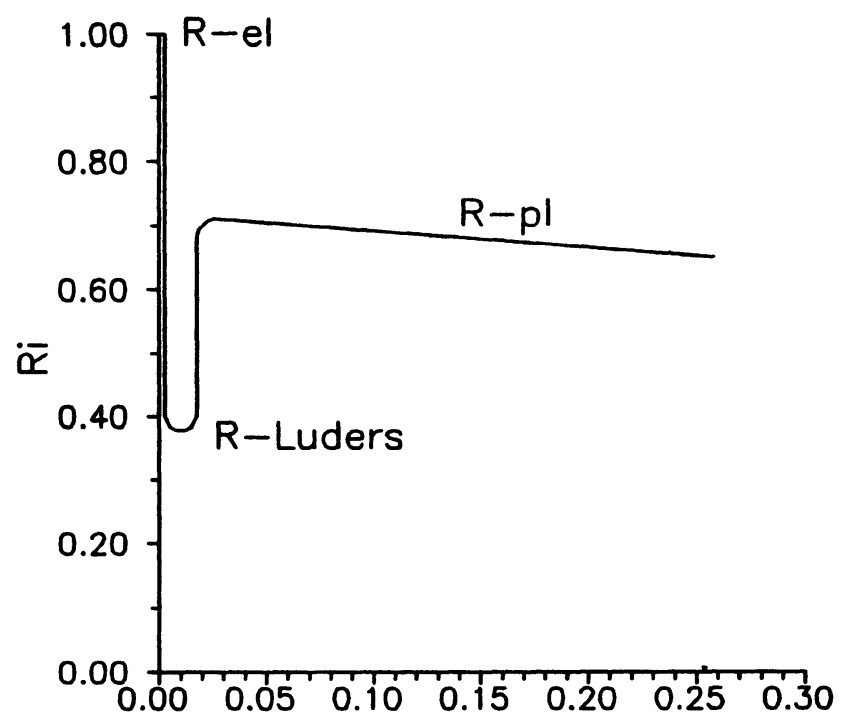

Figure 13 Schematic illustration of the strain dependence of $r$ in the cases of a) homogeneous and b) inhomogeneous yielding. 
Table 3 Experimentally measured $r$ 's during Lüders band propagation in the experimental alloy.

\begin{tabular}{ccc}
\hline $\begin{array}{c}\text { inclination } \\
\text { angle }\end{array}$ & batch annealed & $\begin{array}{c}\text { continuous } \\
\text { annealed }\end{array}$ \\
RD & 0.4 & 0.4 \\
$15^{\circ}$ & 0.5 & 0.4 \\
$30^{\circ}$ & 0.7 & 0.4 \\
$45^{\circ}$ & 0.5 & 0.4 \\
$60^{\circ}$ & 1.1 & 0.3 \\
$75^{\circ}$ & 1.5 & 1.3 \\
TD & 1.4 & 0.7 \\
\hline
\end{tabular}

\section{Appendix}

Considering that a Lüders band is very narrow, the strain parallel to the band, $\varepsilon_{b}$, is zero. This strain is related to the elongation and width strains by the following relation:

$$
d \epsilon_{b}=d \epsilon_{l} \cos ^{2} \alpha+d \epsilon_{w} \sin ^{2} \alpha=0
$$

where $\alpha$ defines the inclination angle between the band and the loading axis. Defining the contraction ratio $q=-\varepsilon_{w} / \varepsilon_{l}$, the previous equation becomes:

$$
d \epsilon_{l} \cos ^{2} \alpha-q \cdot d \epsilon_{l} \sin ^{2} \alpha=0
$$

The angle $\alpha$ is thus directly related to the contraction ratio:

$$
\alpha=\arctan \left(\sqrt{\frac{1}{q}}\right)
$$

The contraction ratio $q$ being directly related to the $r$-value: $q=r /(1+r)$, the previous equation becomes:

$$
\alpha=\arctan \left(\sqrt{\frac{1+r}{r}}\right)
$$

\section{Acknowledgements}

The authors are indebted to Alcan International for their financial support as well as for supplying aluminum sheets and tensile test samples. They acknowledge with gratitude the financial support received from the Natural Sciences and Engineering Research Council of Canada.

\section{References}

Bunge, H. J. (1970). Some applications of the Taylor theory of polycrystal plasticity. Kristall und Technik, 5, 145-175.

Bunge, H. J. (1974). The effective elastic constants of textured polycrystal in second order approximation. Kristall und Technik, 9, 413-424.

Bunge, H. J. (1982). Texture Analysis in Materials Science. Butterworths, London.

Daniel, D. and Jonas, J. J. (1992). The elastic strain ratio, the Luders strain ratio and the evolution of $r$-value during tensile deformation. Textures and Microstructures, 19, 175-188.

Engler, O., Wagner, P., Savoie, J., Ponge, D. and Gottstein, G. (1993). Strain rate sensitivity of flow stress and its effect on hot rolling texture development. Scripta Metallurgica et Materialia, 28, $1317-1322$. 
MacEwen, S. R., Perrin, R., Green, D., Makinde, A. and Neale, K. W. (1992). An evaluation of planar biaxial deformation in $\mathrm{H} 19$ can-stock sheet. In Proc. 13th Ris $\phi$ International Symposium on Materials Science, Ris $\phi, 539-545$.

Toth, L. S., Gilormini, P. and Jonas, J. J. (1988). Effect of rate sensitivity on the stability of torsion textures. Acta Metallurgica, 36, 3077-3091.

Van Houtte, P. (1991). A method for the generation of various ghost correction algorithms - the example of the positivity method and the exponential method. Textures and Microstructures, 13, 199-212.

Welch, P. I. Ratke, L. and Bunge, H. J. (1983). Consideration of Anisotropy Parameters in Polycrystalline Metals. Z. Metallkde., 74, 233-237. 